\title{
Pediatric physical therapy and latest developments \& its current status in India
}

\section{Introduction}

Physical therapy or physiotherapy (often abbreviated to PT) is a physical medicine and rehabilitation specialty that by using mechanical force and movements, remediates impairments and promotes mobility, function, and quality of life through examination, diagnosis, prognosis, and physical intervention. Pediatric physical therapists are healthcare professional who are specifically trained to improve the lives and daily function of children who suffer from a wide range of injuries and congenital conditions. The role of a pediatric therapist is to assess and treat the diverse needs of the child to reach maximum potential to function independently and to promote active participation at home, in school, and in the community. Pediatric patients range from infants in the Neonatal Intensive Care Unit to teenage competitive athletes, specifically 0 to 18 years of age and young adults with childhood disorders. The scope has increased considerably in the last few years to decades though India appears to be in its infancy.

\section{History}

Physicians like Hippocrates and later Galen are believed to have been the first practitioners of physical therapy, advocating massage, manual therapy techniques and hydrotherapy to treat people in $460 \mathrm{BC}$. The earliest documented origins of actual physical therapy as a professional group date back to Per Henrik Ling, "Father of Swedish Gymnastics" who founded the Royal Central Institute of Gymnastics (RCIG) in 1813 for manipulation and exercise. ${ }^{1}$ The Swedish word for physical therapist is sjukgymnast: someone involved in gymnastics for those who are ill. In 1887 PTs were given official registration by Sweden's National Board of Health and Welfare. Other countries soon followed. In 1894 four nurses in Great Britain formed the Chartered Society of Physiotherapy. ${ }^{2}$ The School of Physiotherapy at the University of Otego in New Zealand in $1913^{3}$ and the United States 1914 Reed College in Portland, Oregon, which graduated "reconstruction aides. ${ }^{4}$ " Since the profession's inception spinal manipulative therapy has been a component of the physical therapist practice. 5

Modern physical therapy was established towards the end of the 19th century due to events that had an effect on a global scale which called for rapid advances in physical therapy. Soon following American orthopedic surgeons began treating children with disabilities and began employing women trained in physical education and remedial exercise. These treatments were applied and promoted further during the Polio outbreak of 1916. During the First World War women were recruited to work with and restore physical function to injured soldiers and the field of physical therapy was institutionalized. Despite this long history the Pediatric Physiotherapy is still an underdeveloped specialty in India.

What is the role of a pediatric physical therapist?

Pediatric physical therapists are experts in movement analysis and facilitate normal motor development while maximizing functional

\author{
Volume 2 Issue I - 2017 \\ Kanukaushik,' Kishore Kumar ${ }^{2}$ \\ 'Department of Pediatric Physiotherapist \& Pediasuit Therapist, \\ India \\ ${ }^{2}$ Department of Neonatologist \& Pediatrician, Notre Dame \\ University, Australia
}

Correspondence: Kishore Kumar, Department of Neonatologist \& Pediatrician, Notre Dame University, Perth, Australia,Tel + 91 8040202222

Email drkishore@cloudninecare.com

Received: November II, 2016 | Published: March 08, 2017

independence. They promote health and wellness in children in a clinic or community setting by way of screenings or evaluations to identify the focus of intervention. Pediatric physical therapists collaborate with medical, educational, rehabilitation specialists and families to promote optimal physical functioning based on the needs of each child. Physical therapy interventions can facilitate the best cognitive and physical development by addressing flexibility, strength, and energy efficiency with movement in order to optimize play and daily activities.

\section{What conditions can be treated by pediatric physical therapist ${ }^{6}$ ?}

1. Premature babies ( $<36$ weeks gestational age) or low birth weight babies $(<1500 \mathrm{~g})$

2. Newborn babies having difficulty turning their heads

3. Newborn babies not tolerating tummy time

4. Newborn babies having flat spots on the back or side of their heads, especially after 7 weeks old

5. Babies and toddlers who have difficulty with rolling, sitting, crawling and walking

6. Toddlers with pigeon toes, bow legs, in-rolling ankles, knock knees.

7. Children who have difficulties with coordination, balance, walking and running

8. Frequent falls, poor balance and coordination

9. Children and teenagers who have any sports related injuries

10. Children with poor posture or children who complain of frequent muscular pain

11. Some contributing factors to delayed or poor quality motor development are:

A. low muscle tone/strength/endurance 
B. poor or under-developed coordination

C. poor or under-developed balance

D. poor or under-developed core/trunk stability

E. less than ideal body/joint mechanics or alignment

F. communication challenges

G. sensory challenges

Injury-this can include injuries to the musculoskeletal system (i.e. sports injuries) or the neurological system (i.e. traumatic brain injury) the environment.

Awareness of the benefits of PT can be achieved better for health care professionals if it forms part of the medical curriculum in medical schools. ${ }^{7}$ There are many other familiar childhood disorders and diseases that present with movement dysfunction and motor skill issues that can be treated by physical therapy. These include Cerebral Palsy, Autism, Down syndrome, Muscular Dystrophy and associated disorders, Spina Bifida, Juvenile Rheumatoid Arthritis, Cardio-Pulmonary Disorders, Cystic Fibrosis, Cancer, and Traumatic Brain Injury. In addition, many children present with hypotonia and developmental coordination disorder without a formal diagnosis. Orthopedic conditions that can also be treated with PT include scoliosis, back pain, sports injuries, fractures, and orthopedic surgeries. Its benefits have started flowing to many 'functional disorders' like constipation nowadays. ${ }^{8}$

\section{Latest development \& current status of Indian pediatric physical therapist}

Pediatric physical therapists are playing a wonderful role starting from the infants in the Neonatal Intensive Care Unit till young adults with childhood disorders. Globally prematurity is the leading cause of newborn deaths and the second leading cause of death after pneumonia in children under the age of five. According to WHO India is on the top list to have preterm births. Studies have shown remarkable changes in improving preterm death ratio after early intervention therapy and chest physiotherapy involving manual hyperinflation and vibrations, for secretion clearance in NICU. Even children with malnutrition can benefit from PT to improve their muscle bulk while recovering. ${ }^{9}$ Latest advanced therapy known as Pedi suit/Rehab suit Therapy is prerequisite for kids with Cerebral Palsy. This therapy maximizes and develops muscle strength, flexibility and control and minimizes secondary complications such as contractures, fixed deformities, scoliosis, kyphosis etc. Cage therapy and similar therapies are making their appearance in India. ${ }^{10}$ Upcoming role of pediatric physiotherapists in Sports rehabilitation in kids is mesmerizing. A therapist aims to prevent injuries which happens during sports and provides Sport conditioning: balance, proprioception, agility \& coordination training, Postural \& gait re-training, Dynamic core strengthening, Flexibility training according to children needs. Two more unique conditions where pediatric therapist involvement in infants has changed lives:

\section{Head shape management}

1. Flat head and skull asymmetries (plagiocephaly, brachycephaly, scaphocephaly)

2. Assessment and tracking of child's head shape

3. Education on positional treatment specific to child
4. Treatment for neck conditions impacting the position preference

5. Referral to Certified Orthotist for headband/helmet screening and $3 \mathrm{D}$ head scan as required

\section{Torticollis}

i. Tight neck muscle(s) resulting in head twists and or tilt

ii. Education on infant positioning in sleep, play and throughout the day

iii. Hands-on stretching \& strengthening techniques

iv. Age-appropriate play-based exercises

But unfortunately the awareness of the work the physical therapists do is least known leading in many cases to their under utilization. The recent survey done at one of the regional hospitals revealed that $85 \%$ of the non-medical people felt it was just exercises they teach and $50 \%$ of medical professionals thought they do a bit of building weaker muscles. This article helps to highlight the fact that they do more than that. A Pediatric Physical therapist combined with a good Pediatrician, who can refer and monitor the progress of a child-can dream to see all their patients grow up to be healthy, happy and successful.

\section{Acknowledgements}

None.

\section{Conflict of interest}

The author declares no conflict of interest.

\section{References}

1. Bakewell S. Illustrations from the welcome institute library: medical gymnastics and the cyriax collection. Med Hist. 1997;41:487-495.

2. History of the chartered society of physiotherapy. Chartered society of physiotherapy; 2008.

3. Bruce K. History of the school of physiotherapy. School of physiotherapy centre for physiotherapy research; 2007.

4. Reed College. Mission and History; 2008.

5. Bialosky JE, Simon CB, Bishop MD, et al. Basis for spinal manipulative therapy: a physical therapist perspective. $J$ Electromyogr Kinesiol. 2012;22(5):643-647.

6. Baque E, Sakzewski L, Barber L, et al. Systematic review of physiotherapy interventions to improve gross motor capacity and performance in children and adolescents with an acquired brain injury. Brain Inj. 2016;30(8):948-959.

7. Kyriakoulis K, Patelarou A, Laliotis A, et al. Educational strategies for teaching evidence-based practice to undergraduate health students: systematic review. J Educ Eval Health Prof. 2016;3:34.

8. Orhan C, Kara KO, Kaya S, et al. The effects of connective tissue manipulation and Kinesio Taping on chronic constipation in children with cerebral palsy: a randomized controlled trial. Disabil Rehabil. 2016;28:111.

9. Bekele A, Janakiraman B. Physical therapy guideline for children with malnutrition in low income countries: clinical commentary. $J$ Exerc Rehabil. 2016;12(4):266-75.

10. Kaushik K, Kumar RK. Effect of Cage therapy using advanced spider suit compared to traditional physical therapy on gross motor function in children with cerebral palsy-an Indian experience. Int $J$ Neurore habilitation. 2016;3:1. 